\title{
LA-UR-15-28943
}

Approved for public release; distribution is unlimited.

Title: $\quad$ EOS Interpolation and Thermodynamic Consistency

Author(s): $\quad$ Gammel, J. Tinka

Intended for: $\quad$ Report

Issued: 
Disclaimer:

Los Alamos National Laboratory, an affirmative action/equal opportunity employer,is operated by the Los Alamos National Security, LLC for the National NuclearSecurity Administration of the U.S. Department of Energy under contract DE-AC52-06NA25396. By approving this article, the publisher recognizes that the U.S. Government retains nonexclusive, royalty-free license to publish or reproduce the published form of this contribution, or to allow others to do so, for U.S. Government purposes. Los Alamos National Laboratory requests that the publisher identify this article as work performed under the auspices of the U.S. Departmentof Energy. Los Alamos National Laboratory strongly supports academic freedom and a researcher's right to publish; as an institution, however, the Laboratory does not endorse the viewpoint of a publication or guarantee its technical correctness. 


\section{EOS Interpolation and Thermodynamic Consistency}

\section{J. Tinka Gammel, T-1, LANL}

10/7/11, modified 11/13/15 to add Air example (Fig. 3)

As discussed in LA-UR-08-05451 [1], the current interpolator used by Grizzly, OpenSesame, EOSPAC, and similar routines is the rational function interpolator from Kerley [2]. While the rational function interpolator is well-suited for interpolation on sparse grids with logarithmic spacing and it preserves monotonicity in 1-d, it has some known problems:

1) Thermodynamic quantities are interpolated separately, rather than in a thermodynamically consistent manner, which can lead to thermodynamic inconsistencies.

2) While monotonicity is preserved for the 1-d form, for either of the 2-d interpolation formulas given in [2], regions with monotonic grid values can have non-monotonic interpolants within the 2-d grid.

3) The 2-d interpolation is $\mathrm{C}^{0}$ (or $\mathrm{C}^{1}$ for the form given in the appendix), and so thermodynamic quantities calculated from the interpolated values can have unphysical discontinuities.

\section{Form of the 1-d Rational Function Interpolator}

Re-analyzing Kerley's 1-d form, one sees it can be re-written as:

$\mathrm{f}[\mathrm{q}]=+\mathrm{f0} *(1-\mathrm{q})+\mathrm{f} 1 * \mathrm{q}+(\mathrm{df0}-\mathrm{f} 1+\mathrm{f0}) * \mathrm{~g}[\mathrm{q}, \mathrm{dg} 1]$

where for Kerley's form $\mathrm{g}$ is given by

$$
\begin{aligned}
& \mathrm{g}_{\mathrm{K}}[\mathrm{q}, \operatorname{dg} 1]=\operatorname{dg} 1^{*}(1-\mathrm{q})^{*} \mathrm{q} /(\mathrm{dg} 1 *(1-\mathrm{q})-\mathrm{q}) \quad \text { for } \operatorname{dg} 1<0 \\
& \mathrm{~g}_{\mathrm{K}}[\mathrm{q}, \operatorname{dg} 1]=\operatorname{dg} 1 * \mathrm{q} *(1-\mathrm{q}) *(1-2 * \mathrm{q}) /(\operatorname{dg} 1 *(1-\mathrm{q})+\mathrm{q}) \quad \text { for } \operatorname{dg} 1>0
\end{aligned}
$$

and where

$$
\mathrm{q}=(\mathrm{x}-\mathrm{x} 0) /(\mathrm{x} 1-\mathrm{x} 0)
$$

$\mathrm{del}=(\mathrm{x} 1-\mathrm{x} 0)$

$\mathrm{df} 0=\mathrm{df} / \mathrm{dq}\left|\mathrm{x} 0=\mathrm{del}{ }^{*} \mathrm{df} / \mathrm{dx}\right| \mathrm{x} 0$

$\mathrm{df} 1=\mathrm{df} / \mathrm{dq}|\mathrm{x} 1=\mathrm{del} * \mathrm{df} / \mathrm{dx}| \mathrm{x} 1$

$\mathrm{dg} 1=(\mathrm{df} 1-\mathrm{f} 1+\mathrm{f} 0) /(\mathrm{df} 0-\mathrm{f} 1+\mathrm{f} 0)$

and where $\mathrm{f} 0, \mathrm{fl}$ are the tabulated values of the function at pts $\mathrm{x} 0, \mathrm{x} 1$, and $\mathrm{df} / \mathrm{dx} \mid \mathrm{x} 0,1$ are it's estimated derivatives at these points. Note g satisfies the symmetry condition

$\mathrm{g}[\mathrm{q}, \mathrm{dg} 1]=-\operatorname{dg} 1 * \mathrm{~g}[1-\mathrm{q}, 1 / \mathrm{dg} 1]$

so the interpolant is direction-independent, and it's behavior is spanned by analyzing $-1<\operatorname{dg} 1<1$.

\section{Thermodynamic consistency}

From the re-analysis above of Kerley's form one can easily see that for, eg, interpolation of a (Helmholtz free E) as a function of density, instead of using estimated derivates one could use the tabulated values of $p$ (pressure) and $\mathrm{da} / \mathrm{drho}=\mathrm{p} / \mathrm{rho}^{\wedge} 2$. The resultant behavior of a and $\mathrm{p}$ was shown in Fig 5.a of [1] for Al 3720, reproduced here as Fig. 1.

From Fig 1, one sees clearly that there are problems with the tabulated EOS. There are likely several sources of the problem [3]:

1) numerical issues with TFD,

2) replacing some gridlines with their interpolants, and

3 ) thermodynamically inconsistent splicing of model regions.

These issues are not specific to this EOS, and these problems are more significant on denser EOS grids. Analyzing the values, it turns out that the minimum and maximum monotonic $\mathrm{p}$ interpolants $(\mathrm{p}=\mathrm{pmin}$ and $\mathrm{p}=\mathrm{pmax}$ ) simply do allow for any monotonic interpolant which satisfies

a1-a0 $=$ int $\{$ rho0,rho1 $\}$ drho $\mathrm{p}($ rho $) /$ rho $^{\wedge} 2$

This indicates there is a need for a quick graphic test to highlight areas where EOS's have problems with thermodynamic consistency for their tabulated values (independent of the interpolation scheme).

Fig. 6 of [1], reproduced here as Fig. 2, showed plots for the Al 3720 EOS highlighting values for the following two tests:

1) a,p consistency:

$\left\{\mathrm{a} 1-\mathrm{a} 0-(\mathrm{rho} 1-\mathrm{rho} 0)^{*}\left(\mathrm{p} 1 / \mathrm{rho}^{\wedge} 2+\mathrm{p} 0 / \mathrm{rho}^{\wedge} 2\right) / 2\right\} /\left\{(\mathrm{rho} 1-\mathrm{rho} 0)^{*}\left(\mathrm{p} 1 / \mathrm{rho}^{\wedge} 2-\mathrm{p} 0 / \mathrm{rho} 0^{\wedge} 2\right) / 2\right\}$

2) a,s consistency:

$\{\mathrm{a} 1-\mathrm{a} 0-(\mathrm{t} 1-\mathrm{t} 0) *(-\mathrm{s} 1-\mathrm{s} 2) / 2\} /\{(\mathrm{t} 1-\mathrm{t} 0) *(-\mathrm{s} 1+\mathrm{s} 0) / 2\}$ 
Values of these quantities with magnitudes greater than 1 emphasize regions of the grid where a minimum or maximum in $\mathrm{p}$ or s, respectively, would be required to fit both tabulated values, and so no thermodynamically consistent monotonic fit is possible for that line segment. Although a few of the highlighted regions are where $\mathrm{p}$ or $\mathrm{s}$ are expected thermodynamically to have an extremum, there is significant "stipple" indicating the tabulated points are thermodynamically inconsistent - again, independent of the interpolation scheme.
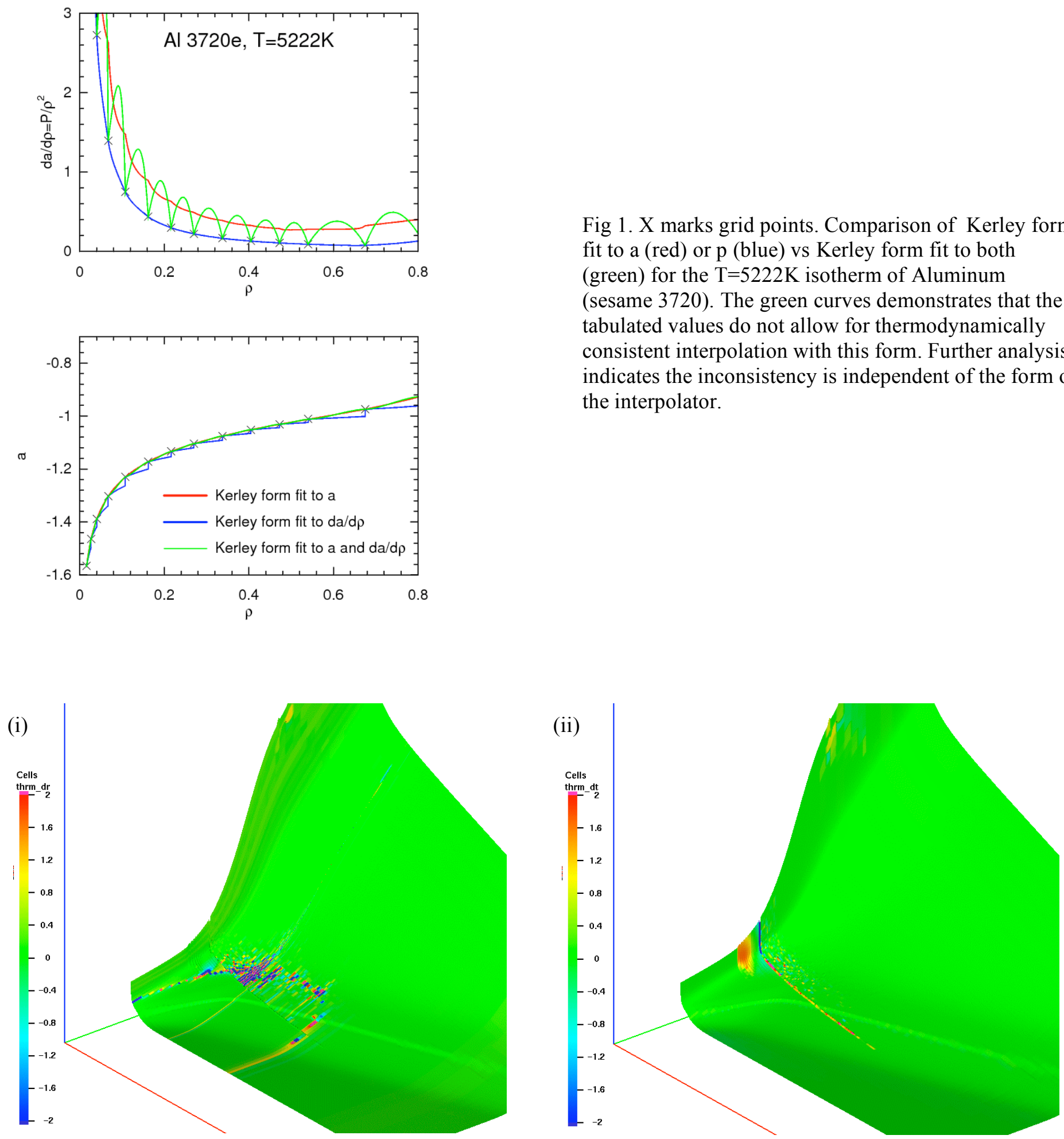

Fig 2. BeO EOS (sesame 7613 with TFD contributions having KGH's corrections) colored to highlight regions (nongreen) where the tabulated values of (i) p,a (left) and (ii) p,s (right) require p or s, respectively, to have an extremum. $\mathrm{x}, \mathrm{y}, \mathrm{z}$ values for these plots are $\mathrm{r}, \mathrm{t}, \mathrm{s}$ While not easily visible at this scale, blowing up the $\mathrm{p}$, a relation plot shows a line of inconsistency near rho=rhoref, an artifact of the very dense grid used in that region.

Fig 1. X marks grid points. Comparison of Kerley form fit to a (red) or p (blue) vs Kerley form fit to both (green) for the $\mathrm{T}=5222 \mathrm{~K}$ isotherm of Aluminum (sesame 3720). The green curves demonstrates that the tabulated values do not allow for thermodynamically consistent interpolation with this form. Further analysis indicates the inconsistency is independent of the form of the interpolator.

(ii) 
Generalizing the tests discussed in [1] and above, once can define monotonicity consistency test for relations of form $\mathrm{V}=\mathrm{dW} / \mathrm{dX} \mid \mathrm{Y}$ where $\mathrm{V}, \mathrm{W}$ combinations of tabulated t,rho,p,e,a and $\mathrm{X}, \mathrm{Y}=\mathrm{t}$, rho or rho,t Specifically, define

test_mono $=(\mathrm{W} 1-\mathrm{W} 0-(\mathrm{X} 1-\mathrm{X} 0) *(\mathrm{~V} 1+\mathrm{V} 0) / 2) /((\mathrm{X} 1-\mathrm{X} 0) *(\mathrm{~V} 1-\mathrm{V} 0) / 2)$ where

$\mathrm{W} 1=\mathrm{W}(\mathrm{t}=\mathrm{t} 1, \mathrm{rho}=\mathrm{rho} 1)$, etc and $\mathrm{Y} 0=\mathrm{Y} 1$

then if $\mathrm{V}(\mathrm{X})$ is monotonic, |test_mono $\mid<1$ if $\mathrm{V}$ and $\mathrm{W}$ are consistent.

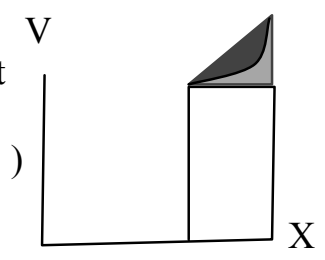

$0 \quad 1$

Test compares area needed for integral minus linear fit area (dark grey) to shaded triangle (light + dark grey). $\mathrm{V}(\mathrm{X})$ must lie in triangle if monotonic (convex case shown).

Non-monotonic fits can also satisfy $\mid$ test_mono $\mid<1$, so this only tests if a

Once can also define smoothness consistency test for relations of same form.

Specifically, define

$\mathrm{dWdX} \min =\min [(\mathrm{W} 1-\mathrm{W} 0) /(\mathrm{X} 1-\mathrm{X} 0),(\mathrm{W} 2-\mathrm{W} 1) /(\mathrm{X} 2-\mathrm{X} 1)]$

montonic fit is possible for the given tabulated values.

dWdX_max $=\max ["]$

test $\_$smooth $=\left(\mathrm{V} 1-\left(\mathrm{dWdX} \_\min +\mathrm{dWdX} \_\max \right) / 2\right) /\left(\left(\mathrm{dWdX} \_\max -\mathrm{dWdX}\right.\right.$ min $\left.) / 2\right)$

then $\mid$ test_smooth $\mid<1$ if $\mathrm{V}$ at point 1 lies between discrete slopes of $\mathrm{W}$ on either side of

that point and $\mathrm{V}$ and $\mathrm{W}$ are consistent.

Specific cases of these tests of interest for SESAME EOS tables and the variables names as currently coded to plot as 3-d gmv files are given below and shown in Fig.s 2 and 3.

a,p:

Using the relation $\mathrm{da} / \mathrm{drho} \mid \mathrm{t}=\mathrm{p} / \mathrm{rho}^{\wedge} 2$ yields

W

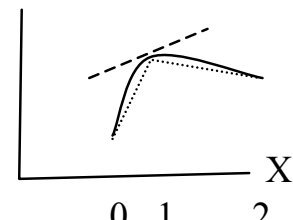

satisfying smoothness assumption that $\mathrm{dW} / \mathrm{dX}$ lies between discrete slopes doesn't rule out nonmonotonic regions.

smoothness, monotonicity tests with: $\mathrm{W}=\mathrm{a}, \mathrm{V}=\mathrm{p} / \mathrm{rho}^{\wedge} 2 \mathrm{X}=\mathrm{rho} \mathrm{Y}=\mathrm{t}$.

This is plotted as "thrm_dr" for the BeO EOS 7613 in Figure (2.i), with

thrm_dr $=\left\{\mathrm{a} 1-\mathrm{a} 0-(\right.$ rhol-rho0 $\left.) *\left(\mathrm{p} 1 / \mathrm{rho}^{\wedge} 2+\mathrm{p} 0 / \mathrm{rho}^{\wedge} 2\right) / 2\right\} /\left\{(\text { rho1-rho0 })^{*}\left(\mathrm{p} 1 / \mathrm{rho} 1 \wedge 2-\mathrm{p} 0 / \mathrm{rho} 0^{\wedge} 2\right) / 2\right\}$

a,s (a,e):

Using the relation $\mathrm{da} / \mathrm{dt}=-\mathrm{s}=(\mathrm{a}-\mathrm{e}) / \mathrm{t}$ yields

smoothness, monotonicity tests with: $\mathrm{W}=\mathrm{a}, \mathrm{V}=(\mathrm{a}-\mathrm{e}) / \mathrm{t}, \mathrm{X}=\mathrm{t} \mathrm{Y}=$ rho

This is plotted as "thrm_dt" for the $\mathrm{BeO}$ EOS 7613 in Figure (2.ii), with

thrm_dt $=\{\mathrm{a} 1-\mathrm{a} 0-(\mathrm{t} 1-\mathrm{t} 0) *(-\mathrm{s} 1-\mathrm{s} 2) / 2\} /\{(\mathrm{t} 1-\mathrm{t} 0) *(-\mathrm{s} 1+\mathrm{s} 0) / 2\}$

Using the relation $u=-\left(T^{\wedge} 2\right) d(a / T) / d T \mid v$ yields

smoothness, monotonicity tests with: $\mathrm{W}=\mathrm{a} / \mathrm{T}, \mathrm{V}=-\mathrm{u} / \mathrm{t} \wedge 2 \mathrm{X}=\mathrm{t} \mathrm{Y}=\mathrm{rho}$

p,e:

using the relation

$\mathrm{P}=\mathrm{T}^{*} \mathrm{dP} / \mathrm{dT}\left|\mathrm{rho}+\mathrm{rho}^{\wedge} 2 * \mathrm{du} / \mathrm{drho}\right| \mathrm{T}$

and defining

dpdt $01=(\mathrm{p} 1-\mathrm{p} 0) /(\mathrm{t} 1-\mathrm{t} 0)$

dpdt $12=(\mathrm{p} 2-\mathrm{p} 1) /(\mathrm{t} 2-\mathrm{t} 1)$

dudr $01=(\mathrm{u} 1-\mathrm{u} 0) /(\mathrm{t} 1-\mathrm{t} 0)$

dudr $12=(\mathrm{u} 2-\mathrm{u} 1) /(\mathrm{t} 2-\mathrm{t} 1)$

p_0101 $=\mathrm{t} 1 *$ dpdt_01 + rho $^{\wedge} 2 *$ dudr_01

p_1201 $=\mathrm{t} 1 * \mathrm{dpdt} \_12+\mathrm{rho}^{\wedge} 2 * \mathrm{dudr}_{-} 01$

p_0112 $=\mathrm{t} 1 *$ dpdt_01 + rho $^{\wedge} 2 *$ dudr_12

p_min $=\min ($ p_0101,p_0112,p1201,p1212)

p_av $=\left(p \_\max +p \_\min \right) / 2$

p_1212 $=\mathrm{t} 1 *$ dpdt_12 + rho $^{\wedge} 2 *$ dudr_12

p_max $=\max \left(\mathrm{p} \_0101, \mathrm{p} \_0112, \mathrm{p} 1201, \mathrm{p} 1212\right)$

thrm_du $=\left(\mathrm{p}-\mathrm{p} \_\right.$min $) / \mathrm{d} \_\mathrm{p}$

d_p $=($ p_av-p_min $)$

Then $\mid$ thrm_du $\mid<1$ if $\mathrm{dP} / \mathrm{dT} \mid$ rho and du/drho $\mid \mathrm{T}$ at a given point lie between the discrete slopes on either side of

$\mathrm{P}(\mathrm{t}, \mathrm{rho}=\mathrm{const})$ and $\mathrm{u}(\mathrm{t}=$ const, rho $)$, respectively.

This is plotted as "thrm_du" for the Air EOS 5030 in Figure (3), with thrm_du as defined above.

Additional cases can relatively easily be added to the variables plotted should there be sufficient interest. 


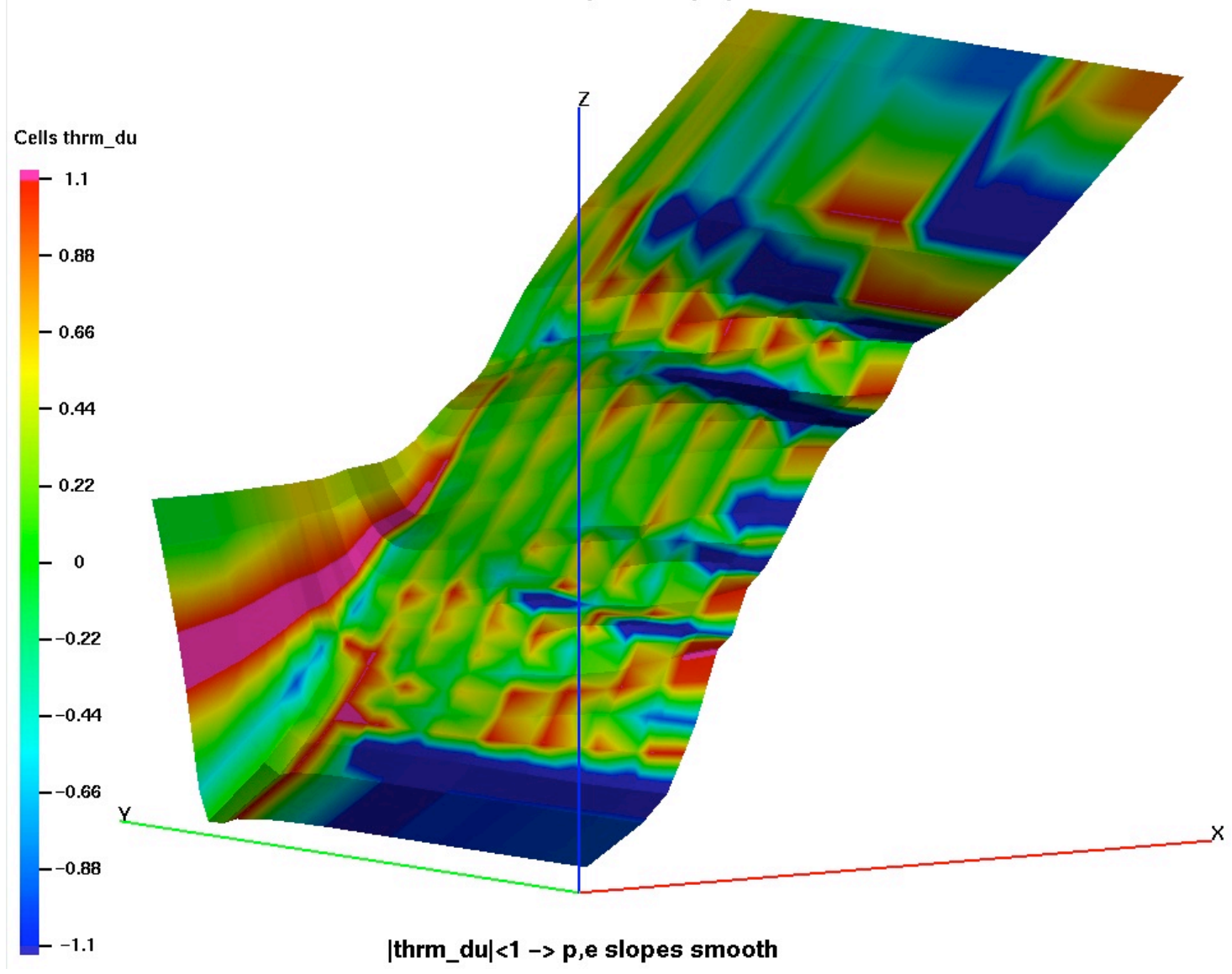

Fig. 3: $u(t, r h o)$ for Air EOS (sesame 5030) colored to highlight regions (non-green) where the tabulated values of $\mathrm{p}, \mathrm{u}$ require at least one of the derivatives $\mathrm{dP} / \mathrm{dT} \mid$ rho and $\mathrm{du} / \mathrm{drho} \mid \mathrm{T}$ to have a local extremum. The plot xyz corresponding to $\mathrm{x}=\mathrm{x}^{\prime} / \max \left|\mathrm{x}^{\prime}\right|$ with $\mathrm{x}^{\prime}=\operatorname{asinh}(\mathrm{t} / \mathrm{RT}), \mathrm{y}=\mathrm{y}^{\prime} / \max \left|\mathrm{y}^{\prime}\right|$ with $\mathrm{y}^{\prime}=\operatorname{asinh}\left(\mathrm{r} /\left(1 . \mathrm{d}-4^{*}\right.\right.$ rhoref $)$ ), and $\mathrm{z}=\mathrm{z}^{\prime} / \max \left|\mathrm{z}^{\prime}\right|$ with $\mathrm{z}^{\prime}=\operatorname{asinh}(\mathrm{e} / .5)$. Note however this analysis assumes the contribution to the total EOS of the chemical potential can be ignored as at fixed particle number. However, since 5030 is a multiphase EOS, with the percentage of each species varying [4], this "effective single phase" consistency test should be re-examined in the grand canonical ensemble.

\section{References}

[1] J.T. Gammel, LA-UR-08-05451

[2] G.I. Kerley, LA-6903-MS

[3] S. Crockett, private communication.

[4] H.C. Grabosky, UCID-16901 